
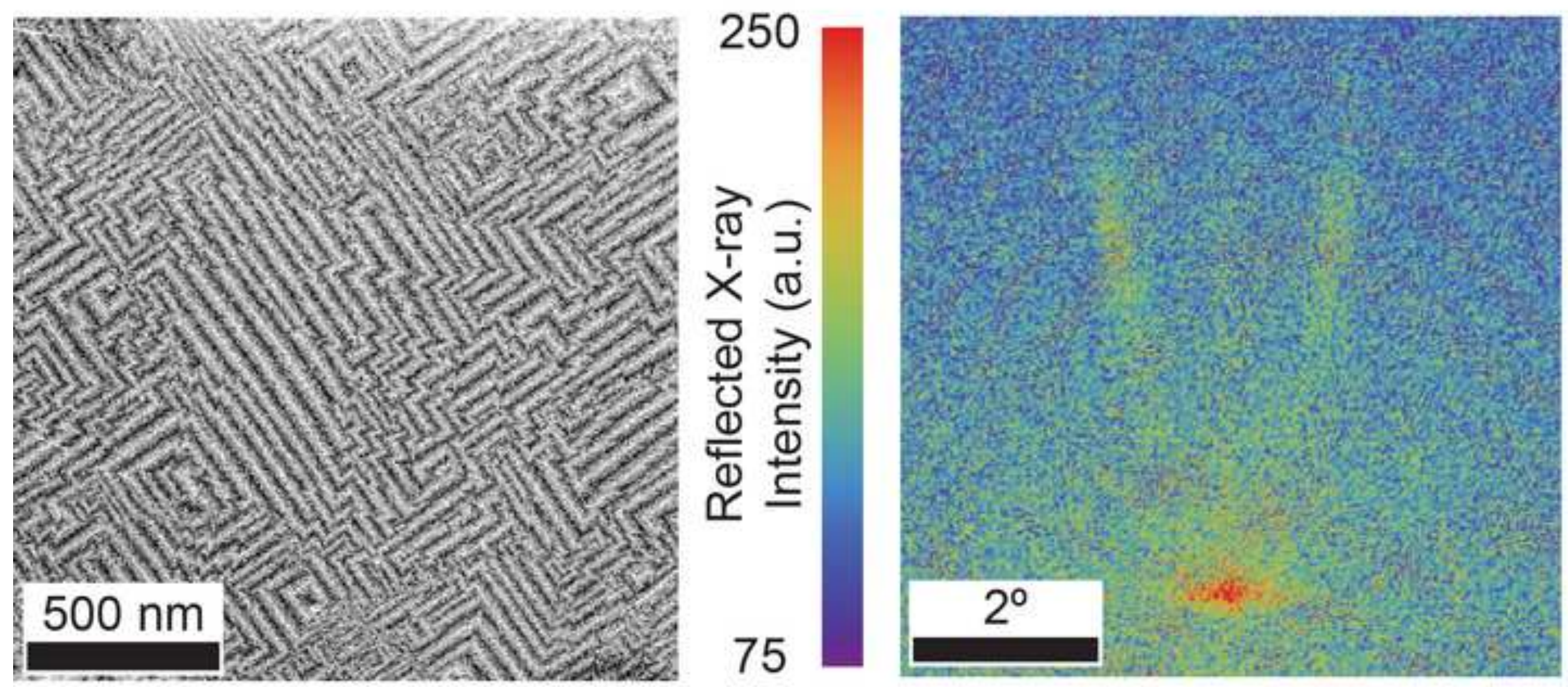


\section{Graphene-induced Ge (001) surface faceting}

Kyle M. McElhinny, Robert M. Jacobberger, Alexander J. Zaug, Michael S. Arnold, and Paul G. Evans $^{\mathrm{a}}$

Department of Materials Science and Engineering, University of Wisconsin-Madison, Madison, Wisconsin 53706, USA.

${ }^{\text {a }}$ Electronic mail: pgevans@wisc.edu.

Faceted Ge surfaces result from the growth of a graphene overlayer on Ge (001) by chemical vapor deposition. The surface exhibits four-fold symmetry after faceting, with the surface normal of the facets tilted towards [100] from the average surface normal. X-ray reflectivity measurements allow the facet angles, directions, and symmetry to be measured precisely as a function of deposition conditions. Graphene grown from a $\mathrm{CH}_{4}$ precursor in $\mathrm{a}_{2} / \mathrm{Ar}$ carrier atmosphere at temperatures from 870 to $920^{\circ} \mathrm{C}$ yields facets on the Ge surface with an average facet angle of $7.70^{\circ} \pm 0.07^{\circ}$. Additionally, a distribution of facet angles is observed with an angular spread of approximately $\pm 1^{\circ}$. The facet pattern has four-fold symmetry over a large area with no indication of the formation of competing facets in reflectivity measurements. The facet angle tends toward the $\{107\}$ facet of Ge with slight variation as a function of temperature indicating that the facet angles are dominated by surface energetics. The slight dependence on temperature is accompanied by a reconstruction of the surface into $\{001\}$ facets under slowcooling conditions, suggesting that the surface diffusion kinetics and temperature dependence have an important role in the formation of the faceted surface structure at lower temperatures. 


\section{Introduction}

The formation of surface facets provides insight into the chemistry and energetics of crystal surfaces and their interfaces with other materials. The faceting of an initially planar surface occurs through a process of nanometer scale self-assembly that exposes new crystal faces and creates additional surface area [1]. While surface faceting is ultimately driven by a minimization of the total surface free energy, the evolution of the surface upon, for example, the creation of interfaces, is controlled by kinetic processes including nucleation and diffusion [1]. By studying the surface geometry and processing conditions that lead to faceting, it is possible to examine the interplay between thermodynamics and kinetics in complex interfacial systems. Although faceting phenomena have most often been observed with atomic or molecular absorbates, recent observations have found that faceting can also occur during the formation of two-dimensional materials.

In particular, the formation of Ge facets below graphene grown on Ge (001) via chemical vapor deposition was recently reported [2]. These Ge facets form during the deposition of graphene at elevated temperatures and are stable under ambient conditions after rapid cooling to room temperature [2]. The faceting is highly selective, as it only occurs in areas where graphene has nucleated and is even observed below relatively small crystals with dimensions less than 10 nm. Atomic force microscopy shows that the Ge/graphene faceting pattern exhibits four-fold symmetry, with approximately equal area occupying each of the four facet domains [2]. The nanometer-scale structure of the faceted Ge/graphene surface is clear from scanning tunneling microscopy studies, which show that the facets are stable under ambient conditions and upon reintroduction into vacuum [2]. These Ge facets belong to the $\{10 \mathrm{~L}\}$ family. Interestingly, such faceting was not reported in other studies of graphene growth on Ge (001), which may be due to 
differences in growth conditions, differences in the thermal profile, or the absence of characterization of the Ge surface $[3,4]$.

The formation of $\{10 \mathrm{~L}\}$ facets has been previously observed on Ge surfaces under a number of conditions. The heteroepitaxial growth of elastically strained Ge with a thickness of a few atomic layers on $\mathrm{Si}(001)$ results in the formation of Ge islands with $\{105\}$ facets, allowing the elastic relaxation of the near-surface region and lowering the total strain energy [5,6]. The Ge $\{107\}$ facet is formed as a result of mounding in Sn-mediated Ge/Ge (001) homoepitaxy [7]. Contributions to the relative energetic stability of facets on Ge include the atomic reconstruction of the faceted surface and the strain dependence of the Ge surface energy $[6,8]$.

Here we report a systematic $\mathrm{x}$-ray reflectivity study of the formation of facets below continuous graphene films grown on Ge (001) during chemical vapor deposition. Unlike atomic force and scanning tunneling microscopy measurements, $\mathrm{x}$-ray scattering measurements provide precise insight into the orientation and roughness of the facets over large areas and thus provide precise statistical averages for the faceted surface structure. The creation of multiple surfaces with different orientations splits the surface diffraction spots in surface x-ray diffraction and reflectivity, producing multiple crystal truncation rods [9]. For an accurately oriented high-index surface, each truncation rod passes through both the origin of reciprocal space and a series of Bragg reflections. With small misorientations, the origin and each Bragg reflection produce a rod of intensity at a specular angle depending on the misorientation [9]. At the small angles of x-ray incidence on which we focus here, the specular reflection from the surface is thus split by the pattern of facets [10]. In the limit of the x-ray beams with short transverse coherence lengths produced by laboratory $\mathrm{x}$-ray sources, the scattering pattern of surfaces with multiple facets can be accurately approximated as the appropriately weighted sum of the intensities of a series of 
misoriented surfaces. X-ray reflectivity studies of faceted surfaces have included step-driven faceting in $\mathrm{Si}$ [11-13], faceting in $\mathrm{Au}$ [14] and $\mathrm{Pt}$ [15], and earlier studies on $\mathrm{Cu}$ surfaces [10]. In a technical sense, the results reported here extend x-ray reflectivity methods to the special case of Ge/graphene and describe the analysis methods necessary to perform such studies using large two-dimensional x-ray detectors.

Understanding the surface energetics and interactions of the Ge/graphene system is also important to advance the synthesis of graphene on semiconducting substrates, and in ultimately promoting better compatibility of graphene with conventional microelectronics [2,3]. The formation of facets during the synthesis of graphene nanoribbons on Ge (001) is particularly interesting, as this growth process has the potential to lead to arrays of high-quality semiconducting nanoribbons directly on a substrate compatible with conventional microelectronics. An improved understanding of the thermodynamics and kinetics of the Ge/graphene system will help lead to increased structural control during the creation of graphene nanoribbon arrays and continuous graphene sheets, and will enable expansion of this insight to growth procedures for other monolayer/substrate combinations.

\section{Experimental Conditions}

The faceting of the Ge surfaces was investigated using Ge/graphene samples grown at temperatures of $870{ }^{\circ} \mathrm{C}, 910{ }^{\circ} \mathrm{C}$, and $920{ }^{\circ} \mathrm{C}$ at atmospheric pressure using $4.6 \mathrm{sccm}$ of $\mathrm{CH}_{4}$ as the carbon precursor and a mixture of $200 \mathrm{sccm}$ of $\mathrm{Ar}$ and $100 \mathrm{sccm}$ of $\mathrm{H}_{2}$ as the reducing carrier gas. Growth times of 42,12 , and 10 hours were used for synthesis at $870{ }^{\circ} \mathrm{C}, 910{ }^{\circ} \mathrm{C}$, and 920 ${ }^{\circ} \mathrm{C}$, respectively, to ensure that a continuous layer of graphene covered the entire surface. Following growth, the samples were rapidly cooled in the same environment used during 
synthesis by sliding the furnace away from the growth zone to bring the samples to room temperature. This process resulted in complete coverage of the surface by a single layer of graphene distributed across sharp hill-and-valley facets [2]. Figure 1(a) and (b) show SEM and AFM images, respectively, of the local surface faceting of the Ge under the graphene. The dimensions of the faceted structure visible in the SEM and AFM images are typically less than 6 $\mathrm{nm}$ high and nearly $70 \mathrm{~nm}$ wide, valley-to-valley, along their short axis. The ridges between facets are nearly parallel to the [100] and [010] directions. The faint ripples visible in the AFM image in Fig. 1(b) arise from wrinkling of the graphene during cooling as a result of the mismatch in the thermal expansion coefficient between the graphene and the Ge. Interestingly, graphene growth followed by a much slower cooling rate of $0.5^{\circ} \mathrm{C} / \mathrm{min}$ results in a different $\mathrm{Ge}$ faceting motif, which will be discussed in more detail below.

A systematic precise study of the faceting angles was performed using x-ray reflectivity. The Ge/graphene surface produces specular x-ray reflections from the facets that form during the growth of the graphene layer. Reflectivity measurements can be interpreted with geometric optics in methods similar to previous studies of faceting of Si surfaces $[12,16]$. These methods must be adapted for the use of large-area two-dimensional x-ray detectors that permit the collection of intensity data from multiple surface truncation rods simultaneously.

X-ray reflectivity measurements were performed with a Bruker D8 Discover with a VÅNTEC 500 Area Detector in a theta-theta geometry with the detector subtending an opening angle of $39^{\circ}$. A Bruker $\mathrm{I} \mu \mathrm{S}$ microfocus $\mathrm{x}$-ray source was operated at $50 \mathrm{~W}$ to produce a $\mathrm{Cu} k \alpha$ incident $\mathrm{x}$-ray beam with a flux estimated to be $2.3 \times 10^{7}$ counts per second and a beam divergence of $0.03^{\circ}$. The incident beam had a diameter of $0.5 \mathrm{~mm}$, which was sufficiently small 
to ensure that the incident beam footprint was never larger than the dimensions of the sample, but sufficiently large to gain ensemble information about the faceting.

In x-ray reflectivity measurements of planar surfaces, a theta-two theta scan is performed to measure the scattered intensity along the specular rod of reciprocal space with scattered wavevector $\left(0,0, q_{z}\right)$ [17]. The faceted Ge surface presents a more complex x-ray reflectivity problem. We consider the special case in which the footprint of the incident x-ray beam lies along a [100] direction. In this case, two of the facets of the Ge/graphene surface are illuminated at sufficiently small angle to produce specular reflections. Intensities from these two facets as well as from small areas retaining the (001) orientation lead to the creation of three surface rods simultaneously.

The geometrical arrangement of the x-ray reflectivity measurement is shown in Fig. 1(c). The sample surface orientation is defined as a function of several angles. The surface normal of the average sample surface, denoted $\mathrm{n}_{\mathrm{avg}}$, serves as the $\varphi$ axis. A rotation around $\mathrm{n}_{\mathrm{avg}}$ is denoted by angle $\varphi$. The $\theta$ axis is fixed normal to the $\varphi$ axis. The incident beam is normal to the $\theta$ axis and moves with $\theta$. Thus a right handed coordinate system can be defined in which the $\theta$ axis is in the $\mathrm{x}$ direction, the $\varphi$ axis is in the $\mathrm{y}$ direction, and the $\mathrm{z}$ direction is the beam direction at $\theta=0^{\circ}$. The tilt angle $\tau$ is defined as the angle between the surface normal of the reflecting facet and the phi axis. The $\tau$ axis is normal to the $\varphi$ axis and rotates with $\varphi$. The tilt angle $\tau$ is the sum of the angle of the facets on the surface $\chi$ and a small rotation of the sample around an axis parallel to the beam axis at $\theta=0^{\circ}[12]$. This small misorientation of the sample with respect to the incident beam causes the left and right facets to appear to have slightly different values of $\tau$, an effect which will be discussed further below. The angular locations of the reflections on the x-ray 
detector are measured as angles $\alpha$ and $\beta$, the azimuthal and polar elevation with respect to the incident beam, respectively. $\alpha$ is the angular location of the $\mathrm{x}$-ray reflection measured around an axis normal to the incident beam and in the same plane as the $\phi$ axis. Note that the $\phi$ axis tilts as $\theta$ changes. $\beta$ is the angular location of the reflection measured around a fixed axis coincident with the $\theta$ axis. The values $\alpha=\beta=0$ corresponds to the location of the direct $\mathrm{x}$-ray beam on the detector, indicated schematically by a transmitted beam spot in Fig. 1(c). These angles can in turn be used to determine the orientation of the surface from which the x-rays are reflected. The surface facets are assumed to be oriented approximately along the incident beam footprint with a small azimuthal misorientation $\varphi$ (Fig. 1(c)). With these definitions, the detector angles $\alpha$ and $\beta$ can be expressed as:

$$
\alpha(\theta, \tau, \varphi)=-\tan ^{-1}\left[\frac{2 \cos \varphi \sin \tau(\cos \theta \sin \tau \sin \varphi+\cos \tau \sin \theta)}{-1+2 \cos ^{2} \theta \sin ^{2} \tau \sin ^{2} \varphi+2 \cos ^{2} \tau \sin ^{2} \theta+\sin 2 \tau \sin \varphi \sin 2 \theta}\right]
$$

\section{$\beta(\theta, \tau, \varphi)=\sin ^{-1}[2(\cos \theta \sin \tau \sin \varphi+\cos \tau \sin \theta)(\cos \tau \cos \theta-\sin \tau \sin \varphi \sin \theta)](2)$}

In the case where the azimuthal angle $\varphi$ is zero, such that the direction of the x-ray beam is along the long axis of the facets, the relationship then simplifies to:

$$
\begin{array}{r}
\alpha(\theta, \tau)=\tan ^{-1}\left[\frac{2 \sin \tau \cos \tau \sin \theta])}{1-2 \cos ^{2} \tau \sin ^{2} \theta}\right](3) \\
\beta(\theta, \tau)=\sin ^{-1}\left[2 \cos ^{2} \tau \cos \theta \sin \theta\right](4)
\end{array}
$$

When the small angle approximation is applied to $\theta$ and $\tau$, the relationship further simplifies to:

$$
\alpha(\theta, \tau)=\frac{2 \tau \theta\left(-2+\tau^{2}\right)}{-2+\theta^{2}\left(-2+\tau^{2}\right)^{2}}(5)
$$




$$
\boldsymbol{\beta}(\boldsymbol{\theta}, \tau)=-\frac{1}{\mathbf{4}} \boldsymbol{\theta}\left(-2+\boldsymbol{\theta}^{2}\right)\left(-2+\tau^{2}\right)^{2}(6)
$$

With these relationships, the tilt angle, $\tau$, can be calculated from the position of the reflection on the detector by substituting measured values of $\alpha$ and $\beta$ into equations (3) and (4) and numerically solving the system of equations for $\theta$ and $\tau$.

A detector image and a reflectivity profile extracted along the horizontal direction across the image are shown in Figs. 1(d) and 1(e), respectively. The three intensity maxima in the detector image comprise two reflections from the facets (the maxima to the left and to the right of the center of the image) and a single central reflection from unfaceted regions in which the local surface normal is oriented along the average macroscopic surface normal [12]. In comparison, the reflectivity of the starting, unfaceted Ge surface produces a detector image consisting of a single sharp high-intensity reflection.

\section{Results and Discussion}

The in-plane symmetry of the faceted surface was probed by studying the $x$-ray reflectivity as a function of the azimuthal orientation of the sample. A series of scattering patterns were acquired at values of the azimuthal angle $\varphi$ ranging from $0^{\circ}$ to $360^{\circ}$ with an incident angle $\theta=2^{\circ}$ and exposure times of 30 seconds per pattern. Maxima in the total reflected intensity from a region of interest which includes the scattering from the flat surface and right and left facets are observed at multiples of $90^{\circ}$, as shown in Figure 2(a). The reflection from the flat surface appears throughout the $360^{\circ}$ scan, while the reflection for the right and left facets appears and disappears at $90^{\circ}$ increments. The surface thus has four-fold symmetry, with the facets preferentially oriented close to the $<100>$ directions of the Ge (001) substrate [2]. A 
similar four-fold symmetry is apparent in the SEM and AFM images in Figs. 1(a) and (b). Figure 2(b) shows the intensity of each streak as a function of a very narrow range in $\varphi$. The intensity of each of the individual reflections from the facets varies in this narrow range because the effective incident angle with respect to the facets is changing. Fig. 2(b) was used to determine the alignment of the sample in which the long axis of the facet is precisely parallel to the projection of the X-ray beam on the surface, taking $\varphi=0$ as the point of equal intensities of the two reflections.

Precise measurements of the faceting angles were performed by examining the variation of the angular positions of the reflected beams as a function of the x-ray incident angle. A series of detector images was collected at values of the incident angle $\theta$ from $4^{\circ}$ to $5.5^{\circ}$ in $0.5^{\circ}$ increments. These measurements were conducted at the angle set as $\varphi=0$ in Fig. 2. The value of $\theta$ in this case is far larger than the critical angle for total external reflection, $\theta_{c}=0.32^{\circ}$, and the intensity for each reflected beam is thus several orders of magnitude weaker than the incident beam. The images were acquired using exposure times of 3600 seconds. The series of detector images for the sample grown at $910^{\circ} \mathrm{C}$ is shown in Fig. 3. Two reflections, one from each of the two facets illuminated at small angle at $\varphi=0$, move to higher angles and decrease in intensity as $\theta$ increases. A faint streak of intensity from the flat surface can be observed between the two facet streaks.

The facet angle can be determined from the detector images by examining the dependence of the locations of the intense reflections, $\alpha$ and $\beta$, on the incident angle $\theta$. Figure 4 shows the angles $\alpha$ and $\beta$ associated with the point of maximum intensity of the reflections from the facets of samples grown at different temperatures. Each set of values of $\alpha$ and $\beta$ are used to 
determine the precise values of $\theta$ and $\tau$ using equations (3) and (4) above. The angles listed on Fig. 4 are the total misorientation designated as the tilt angle $\tau$, which includes both the crystallographic angle of the facet and the misorientation of the sample. The measured values of $\alpha$ and $\beta$ and the corresponding fit values of $\theta$ and $\tau$ for each temperature and incident angle are shown in Table 1. The tilt angle $\tau$ is determined for each individual point with an accuracy that systematically increases with increasing $\theta$, ranging from $\Delta \tau=0.4^{\circ}$ at $\theta=4^{\circ}$ to $\Delta \tau=0.3^{\circ}$ at $\theta=5.5^{\circ}$ for the sample grown at $910^{\circ} \mathrm{C}$. The fit curves plotted in figure 4 are calculated as a function of $\theta$ for the given average $\tau$ for each facet.

The values from the individual points from different $\theta$ are averaged to determine the average tilt angle for the right and left facets, which reduces the uncertainty significantly. For example, in the case of the $910^{\circ} \mathrm{C}$ the uncertainty in the average value is $\pm 0.16^{\circ}$. The two values of the tilt angle $\tau$ for the right and left facet are then averaged to determine the average tilt angle for a sample grown at the given temperature. The error in average tilt angle for a given temperature is reduced to $\pm 0.12^{\circ}$.

The average value of $\tau$ for the two facets is plotted in Fig. 5 as a function of the growth temperature. The error bars are computed by propagating the single measurement error. The horizontal dashed lines indicate the facet angle of the nearest $\{10 \mathrm{~L}\}$ facets to the data. A slight variation of the faceting angle with growth temperature is observed. At $870{ }^{\circ} \mathrm{C}$, the facet angle is closest to the $\{108\}$ facet at $7.13^{\circ}$, while the higher temperature points are closer to the $\{107\}$ facet at $8.13^{\circ}$. These are both significantly different than the commonly observed $\{105\}$ facet observed in $\mathrm{Si}-\mathrm{Ge}$ systems [6]. This growth regime $\left(870^{\circ} \mathrm{C}-920^{\circ} \mathrm{C}\right)$ was chosen because it is the regime over which high-quality monolayer graphene growth is achieved, as indicated by Raman 
spectroscopy. Specifically, the Raman D:G ratio was negligible (less than 0.1 ) over this temperature range, indicating a low defect density. The trend in the faceting angle with temperature suggests that while the faceting process is dominated by the energetics of the crystal surface, kinetic processes, such as limiting the growth rate to allow for surface diffusion, still play a role in the formation of facets in this temperature regime.

A further question is whether or not all facet angles are equal or if the angles of the four sets of facets apparent in Fig. 1 are slightly different. For example, a physical difference in angle can arise from the effects of stresses due to sample miscut. If all facet angles are equivalent, however, a difference between the apparent values of $\tau$ can also arise from a small overall misorientaion of the sample with respect to the incident beam. To investigate this, we compare the average value of $\tau$ from the two facets to the $\tau$ value for the reflection from the flat surface for the sample grown at $910^{\circ} \mathrm{C}$. The average value of the right and left facet is $\tau=-0.29^{\circ} \pm 0.12^{\circ}$ compared to $\tau=-0.52^{\circ} \pm 0.21^{\circ}$ for the flat surface. These two values are the same within error, and the angles of the left and right facet are thus identical within the resolution of the measurement. Hence, the average value of $\tau$ for a given sample is the angle $(\chi)$ of the surface facets. The mean value of all of the samples is $7.7^{\circ} \pm 0.07^{\circ}$.

While reflectivity from the starting planar Ge surface appears as a single spot on the xray detector consistent with the incident beam profile, the reflectivity from these faceted surfaces is elongated into streaks of intensity. The length and width of the streaks on the detector provides insight into the angular distribution of the surface facets. In the limiting case of zero roughness, the length of the streaks along their long axis can be explained by a distribution in facet orientations in a combination of the $\theta$ and $\varphi$ angles. By taking the angular derivative of equation 
(4), we find that the angular extent of the streak is twice the change in $\theta$. The sample grown at $910^{\circ} \mathrm{C}$, has an angular width corresponding to a variation in $\theta$ of $0.30^{\circ} \pm 0.02^{\circ}$. Similarly, taking the angular derivative of equation (2) shows that the angular extent of the streak given by $1 / 4$ times the range of $\varphi$. The sample grown at $910{ }^{\circ} \mathrm{C}$ has an angular width corresponding to a variation in $\varphi$ of $2.26^{\circ} \pm 0.17^{\circ}$. Thus, in the limit of zero surface roughness, the elongation of the streaks could be due to rotational disorder of the facets by as large as $0.30^{\circ}$ in $\theta$ or as large as $2.26^{\circ}$ in $\varphi$.

The width of the streaks perpendicular to their long axis can be explained by a distribution in facet orientations in the $\chi$ angles. Since $\chi$ varies with the same relationships as $\tau$, taking the angular derivative of equation (4) shows that the angular width of the streak is 0.136 times the change in $\chi$ at $4^{\circ}$ and 0.188 times the change in $\chi$ at $5.5^{\circ}$. The sample grown at $910{ }^{\circ} \mathrm{C}$ has an angular width corresponding to a variation in $\chi$ of $1.14^{\circ} \pm 0.08^{\circ}$. However, it is unclear whether this distribution arises from variation within the individual facets or from variation between different facets. Additionally, while the model assumes sharp peaks and troughs with flat plateaus, it is possible that a more rounded hill-and-valley structure could account for the observed variation in the facet angle. The presentation of the reflectivity as streaks provides insight into the surface homogeneity over a large length scale.

Table 2 shows the angular widths of the facet reflections as a function of sample growth temperature. As sample growth temperature increases, the distribution of facet orientations narrows, suggesting that the higher growth temperature allows the system to reach a state closer to its thermodynamically favored state. 
Further insight into the interplay between kinetics and thermodynamics at the Ge/graphene interface can be obtained by varying the rate at which the sample is cooled after growth. A SEM image of the Ge surface for a sample cooled at $0.5^{\circ} \mathrm{C} / \mathrm{min}$, is shown in figure 6(a). If a slow cool rate is used, the surface adopts a terraced structure instead of the hill-andvalley structure observed using faster cooling rates. A reflectivity pattern from the terraced structure is shown in Fig. 6(b). The reflectivity pattern shows an elongated streak from the (001) oriented surface and there is no evidence of reflectivity from angled facets. We hypothesize that during the previously discussed, fast cooling process, the facets are quenched into place. However, when a relatively slow cooling rate is used, the temperature decreases below the value at which the $\{107\}$ facets are stable, and the (001) facets are favored. It was previously shown that the faceted Ge surface becomes atomically-flat after annealing at $800^{\circ} \mathrm{C}$ [2]. Thus, we believe that the temperature at which the $\{107\}$ facets becomes more energetically-favorable than the (001) facet is between 800 and $870{ }^{\circ} \mathrm{C}$. It is not yet clear if other transitions exist between these two temperatures. The formation of the terraced structure implies that the faceting is kinetically-limited by surface diffusion. At lower temperatures, the germanium diffusion length during the cooling time is short, leading to the formation of terraces rather than a planar (001) surface.

\section{Conclusion}

This reflectivity study provides insight into the surface energetics and kinetics at the Ge (001)/graphene interface. The reflectivity data indicate the presence of a four-fold symmetric faceted surface structure. The data also provide precise values for the facet angles, which are found to be approximately $7.7^{\circ}$, with the facets being symmetric within experimental error. Additionally, the study provides insight into the lateral homogeneity of the faceted surface, 
yielding an upper limit on the distribution of sample angles found on the surface. The facet angle tends toward the $\{107\}$ facet of Ge with a slight variation with temperature, suggesting that in this temperature regime, while the facet angle under a complete layer of graphene is dominated by surface energetics, the surface diffusion kinetics still play an important role in the formation of the faceted surface structure. This is further demonstrated by the development of a terraced structure at slower cooling rates.

\section{Acknowledgements}

KM and PE acknowledge support from the University of Wisconsin Materials Research Science and Engineering Center (MRSEC) (grant no. DMR-1121288). RJ, AZ, and MA acknowledge support from the DOE Office of Science Early Career Research Program (Grant number DESC0006414) through the Office of Basic Energy Sciences. R.M.J. acknowledges support from the Department of Defense (DOD) Air Force Office of Scientific Research through the National Defense Science and Engineering Graduate Fellowship (No. 32 CFR 168a). The authors also acknowledge use of instrumentation supported by the UW MRSEC (DMR-1121288).

\section{References}

[1] T.E. Madey, W. Chen, H. Wang, P. Kaghazchi, T. Jacob, Nanoscale surface chemistry over faceted substrates: structure, reactivity and nanotemplates, Chem. Soc. Rev. 37 (2008) 2310.

[2] R.M. Jacobberger, B. Kiraly, M. Fortin-Deschenes, P.L. Levesque, K.M. McElhinny, G.J. Brady, et al., Direct oriented growth of armchair graphene nanoribbons on germanium, Nat. Commun. 6 (2015) 8006.

[3] G. Wang, M. Zhang, Y. Zhu, G. Ding, D. Jiang, Q. Guo, et al., Direct Growth of Graphene Film on Germanium Substrate, Sci. Rep. 3 (2013) 2465. 
[4] G. Lippert, J. Dąbrowski, T. Schroeder, M.A. Schubert, Y. Yamamoto, F. Herziger, et al., Graphene grown on Ge(0 0 1) from atomic source, Carbon. 75 (2014) 104.

[5] Y.-W. Mo, B.S. Swartzentruber, R. Kariotis, M.B. Webb, M.G. Lagally, Growth and equilibrium structures in the epitaxy of Si on Si(001), Phys. Rev. Lett. 63 (1989) 2393.

[6] G.-H. Lu, F. Liu, Towards Quantitative Understanding of Formation and Stability of Ge Hut Islands on Si(001), Phys. Rev. Lett. 94 (2005) 176103.

[7] K.A. Bratland, Y.L. Foo, T. Spila, H.-S. Seo, R.T. Haasch, P. Desjardins, et al., Snmediated Ge/Ge (001) growth by low-temperature molecular-beam epitaxy: Surface smoothening and enhanced epitaxial thickness, J. Appl. Phys. 97 (2005) 044904.

[8] O.E. Shklyaev, M.J. Beck, M. Asta, M.J. Miksis, P.W. Voorhees, Role of Strain-Dependent Surface Energies in Ge/Si(100) Island Formation, Phys. Rev. Lett. 94 (2005) 176102.

[9] G. Held, J. Brock, Calculation of x-ray-diffraction profiles from vicinal surfaces, Phys. Rev. B. 51 (1995) 7262.

[10] B. Ocko, S. Mochrie, Reversible faceting of the copper (110) surface: X-ray Fresnel reflectivity, Phys. Rev. B. 38 (1988) 7378.

[11] S. Song, S. Mochrie, G. Stephenson, Faceting Kinetics of Stepped Si(113) Surfaces: A Time-Resolved X-Ray Scattering Study, Phys. Rev. Lett. 74 (1995) 5240.

[12] S. Song, M. Yoon, S.G.J. Mochrie, Faceting, tricriticality, and attractive interactions between steps in the orientational phase diagram of silicon surfaces between [113] and [5512], Surf. Sci. 334 (1995) 153.

[13] S. Song, M. Yoon, S.G.J. Mochrie, G.B. Stephenson, S.T. Milner, Faceting kinetics of stepped Si(113) surfaces: Dynamic scaling and nano-scale grooves, Surf. Sci. 372 (1997) 37.

[14] G. Watson, D. Gibbs, S. Song, A. Sandy, S. Mochrie, D. Zehner, Faceting and reconstruction of stepped Au(111), Phys. Rev. B. 52 (1995) 12329.

[15] G. Watson, D. Gibbs, D. Zehner, M. Yoon, S. Mochrie, Faceting transformations of the stepped Pt(001) surface, Phys. Rev. Lett. 71 (1993) 3166.

[16] S. Song, S.G.J. Mochrie, Attractive step-step interactions, tricriticality, and faceting in the orientational phase diagram of silicon surfaces between [113] and [114], Phys. Rev. B. 51 (1995) 10068.

[17] Jens Als-Nielsen, Des McMorrow, Elements of Modern X-ray Physics, 2nd ed., John Wiley \& Sons, Ltd, 2011. 


\section{Figure Captions}

Figure 1: (a) SEM and (b) AFM images of faceted Ge (001)/graphene. (c) Schematic of the geometry of the x-ray experiment. Reflected x-rays form three distinct spots: one from the right facet, one from the left facet, and one from the flat surface. The geometry of the problem is defined by three angles. The x-ray incident angle relative to the average surface is given by $\theta$. The total angle of the reflecting plane is $\tau$ and $\varphi$ is the angle of azimuthal sample rotation around the average surface normal. The angle $\chi$ defines the angle of the facet with respect to the average surface. The angular location of the reflected $\mathrm{x}$-ray beams is given by the angles $\alpha$ and $\beta$. (d) Xray scattering pattern acquired with an incident angle of $4^{\circ}$, from the Ge/graphene sample grown at $910{ }^{\circ} \mathrm{C}$. The center streak is the $\mathrm{x}$-ray reflection from the portion of the surface aligned with the average surface. The left and right streaks arise from reflections from the left and right facets. (e) Intensity profile taken horizontally across the image in (d).

Figure 2: (a) Azimuthal $(\varphi)$ data showing that the maxima in the total reflected intensity, from a region which includes the reflectivity from the flat surface and right and left facets, are observed at multiples of $90^{\circ}$. The reflection from the flat surface appears throughout the $360^{\circ}$ scan, while the reflection for the right and left facets appears and disappears every $90^{\circ}$. (b) Intensities of the right, left, and center streak as function of the azimuthal angle $\varphi$ over a narrow range. With the assumption that there is equal population of right and left facets, alignment of the x-ray beam with the long axis of the faceting occurs at the point at which the intensities of the scattering from the right and left facets are equal.

Figure 3: Detector images at the indicated incident angles from $\mathrm{Ge} /$ graphene grown at $910^{\circ} \mathrm{C}$. 
Figure 4: Angular positions $\alpha$ and $\beta$ of x-ray reflections for samples grown at (a) $870^{\circ} \mathrm{C}$, (b) $910^{\circ} \mathrm{C}$, and (c) $920^{\circ} \mathrm{C}$. The solid black lines are $\alpha$ and $\beta$ for the total tilt angle $\chi$ of each reflection averaged over all incident angles.

Figure 5: Temperature dependence of facet angles. Horizontal dashed lines are angles of the $\{10$ L) family of crystal facets.

Figure 6: (a) SEM image of Ge surface after slow cooling. In the case of slow cooling, the surface is a terraced structure. (b) X-ray reflectivity pattern from a sample which was slowly cooled. The angled streaks observed from the hill-and-valley structure are absent, as would be expected from a terraced structure composed of a (001) faceted surface.

\section{Table Captions}

Table 1: The measured values of $\alpha$ and $\beta$ and the corresponding fit values of $\theta$ and $\tau$ for each temperature and incident angle.

Table 2: The angular widths of the facet reflections as a function of sample growth temperature. 
McElhinny et al., Figure 1
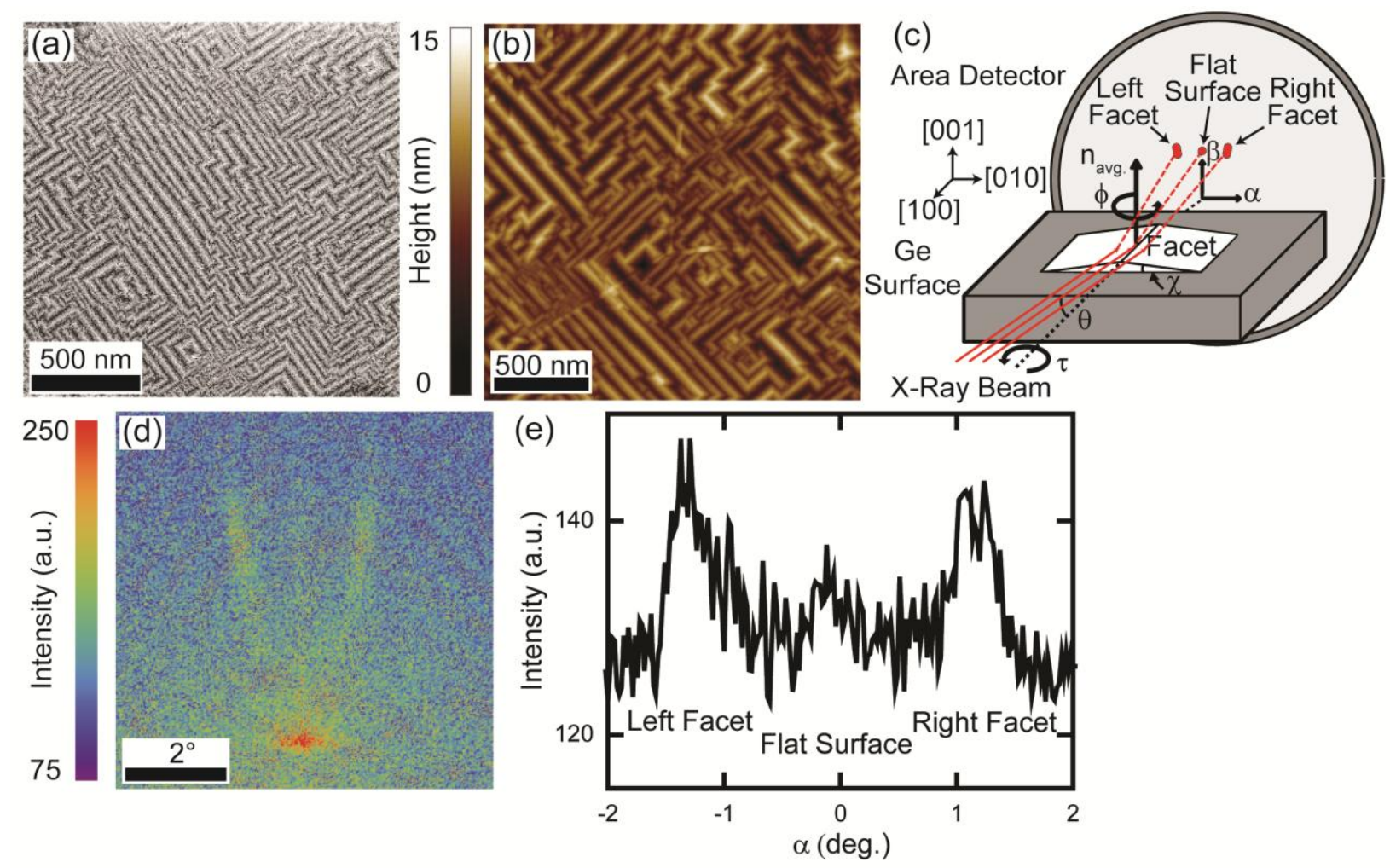
McElhinny et al., Figure 2
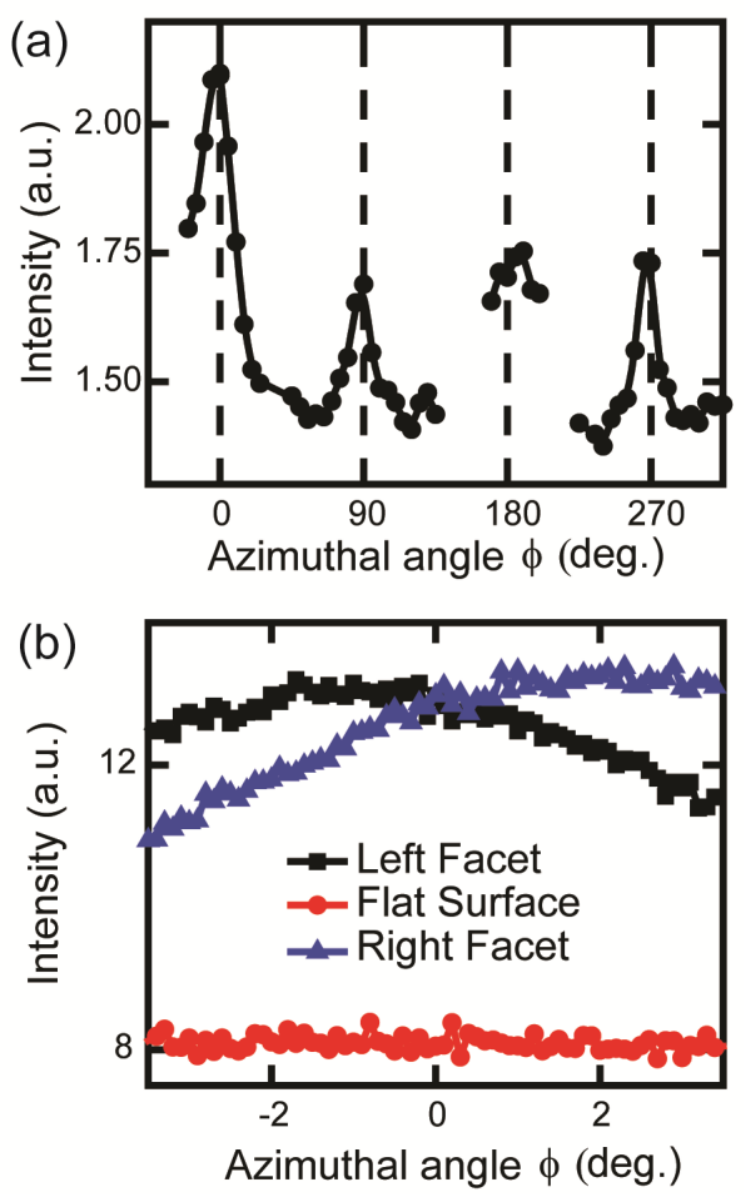
McElhinny et al., Figure 3

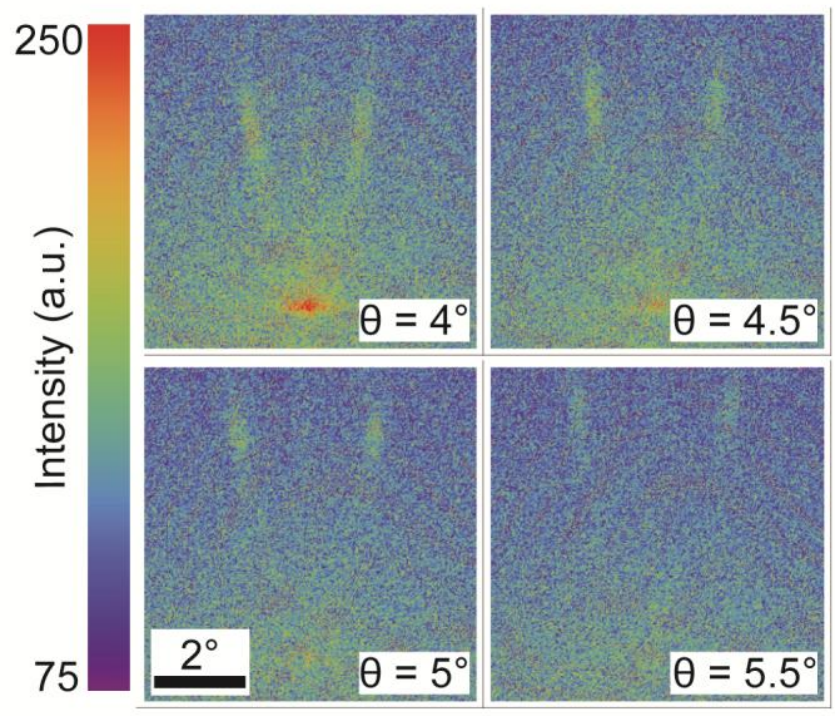




\section{McElhinny et al., Figure 4}
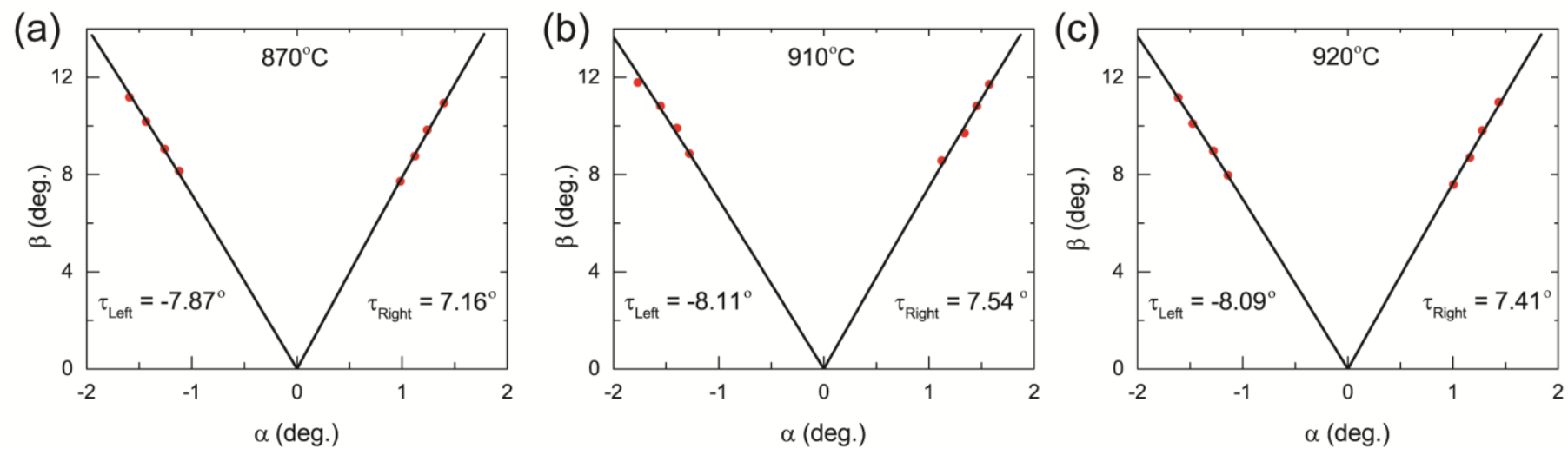
McElhinny et al., Table 1

\begin{tabular}{|c|c|c|c|c|c|}
\hline Growth Temperature & Facet & Measured $\alpha\left({ }^{\circ}\right)$ & Measured $\beta\left({ }^{\circ}\right)$ & Fit $\theta\left({ }^{\circ}\right)$ & Fit $\tau\left({ }^{\circ}\right)$ \\
\hline \multirow{8}{*}{$870^{\circ} \mathrm{C}$} & \multirow{4}{*}{ Left } & -1.12 & 8.15 & 4.15 & -7.76 \\
\hline & & -1.26 & 9.05 & 4.61 & -7.83 \\
\hline & & -1.44 & 10.18 & 5.19 & -7.91 \\
\hline & & -1.59 & 11.18 & 5.70 & -7.97 \\
\hline & \multirow{4}{*}{ Right } & 0.98 & 7.72 & 3.92 & 7.20 \\
\hline & & 1.12 & 8.76 & 4.45 & 7.21 \\
\hline & & 1.24 & 9.83 & 4.99 & 7.08 \\
\hline & & 1.40 & 10.94 & 5.56 & 7.15 \\
\hline \multirow{8}{*}{$910^{\circ} \mathrm{C}$} & \multirow{4}{*}{ Left } & -1.28 & 8.86 & 4.52 & -8.12 \\
\hline & & -1.40 & 9.91 & 5.05 & -7.91 \\
\hline & & -1.55 & 10.82 & 5.52 & -8.04 \\
\hline & & -1.77 & 11.79 & 6.02 & -8.37 \\
\hline & \multirow{4}{*}{ Right } & 1.12 & 8.57 & 4.36 & 7.38 \\
\hline & & 1.34 & 9.70 & 4.94 & 7.75 \\
\hline & & 1.46 & 10.82 & 5.51 & 7.53 \\
\hline & & 1.57 & 11.71 & 5.96 & 7.50 \\
\hline \multirow{8}{*}{$920^{\circ} \mathrm{C}$} & \multirow{4}{*}{ Left } & -1.14 & 7.97 & 4.07 & -8.07 \\
\hline & & -1.28 & 8.97 & 4.58 & -8.02 \\
\hline & & -1.48 & 10.10 & 5.16 & -8.19 \\
\hline & & -1.61 & 11.16 & 5.70 & -8.08 \\
\hline & \multirow{4}{*}{ Right } & 1.00 & 7.59 & 3.86 & 7.47 \\
\hline & & 1.16 & 8.70 & 4.43 & 7.51 \\
\hline & & 1.28 & 9.82 & 4.99 & 7.32 \\
\hline & & 1.44 & 10.97 & 5.58 & 7.33 \\
\hline
\end{tabular}


McElhinny et al., Table 2

\begin{tabular}{|c|c|c|c|}
\hline Growth Temperature & $\underline{\mathbf{8 7 0}^{\circ} \mathbf{C}}$ & $\underline{\mathbf{9 1 0}^{\circ} \mathbf{C}}$ & $\underline{\mathbf{9 2 0}^{\circ} \mathbf{C}}$ \\
\hline Variation in $\theta$ & $0.28^{\circ} \pm 0.01^{\circ}$ & $0.30^{\circ} \pm 0.02^{\circ}$ & $0.22^{\circ} \pm 0.01^{\circ}$ \\
\hline Variation in $\varphi$ & $2.47^{\circ} \pm 0.09^{\circ}$ & $2.26^{\circ} \pm 0.17^{\circ}$ & $1.85^{\circ} \pm 0.11^{\circ}$ \\
\hline Variation in $\chi$ & $1.05^{\circ} \pm 0.11^{\circ}$ & $1.15^{\circ} \pm 0.08^{\circ}$ & $0.85^{\circ} \pm 0.06^{\circ}$ \\
\hline
\end{tabular}


McElhinny et al.,Figure 5

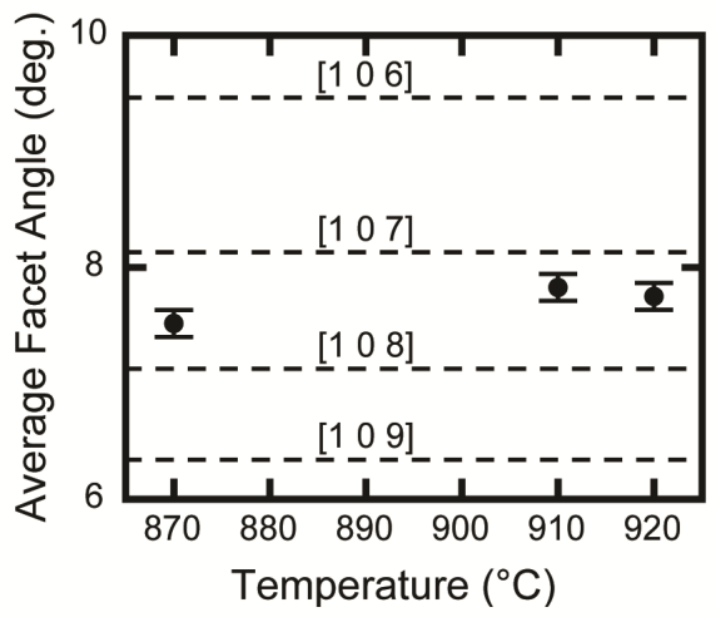


McElhinny et al., Figure 6

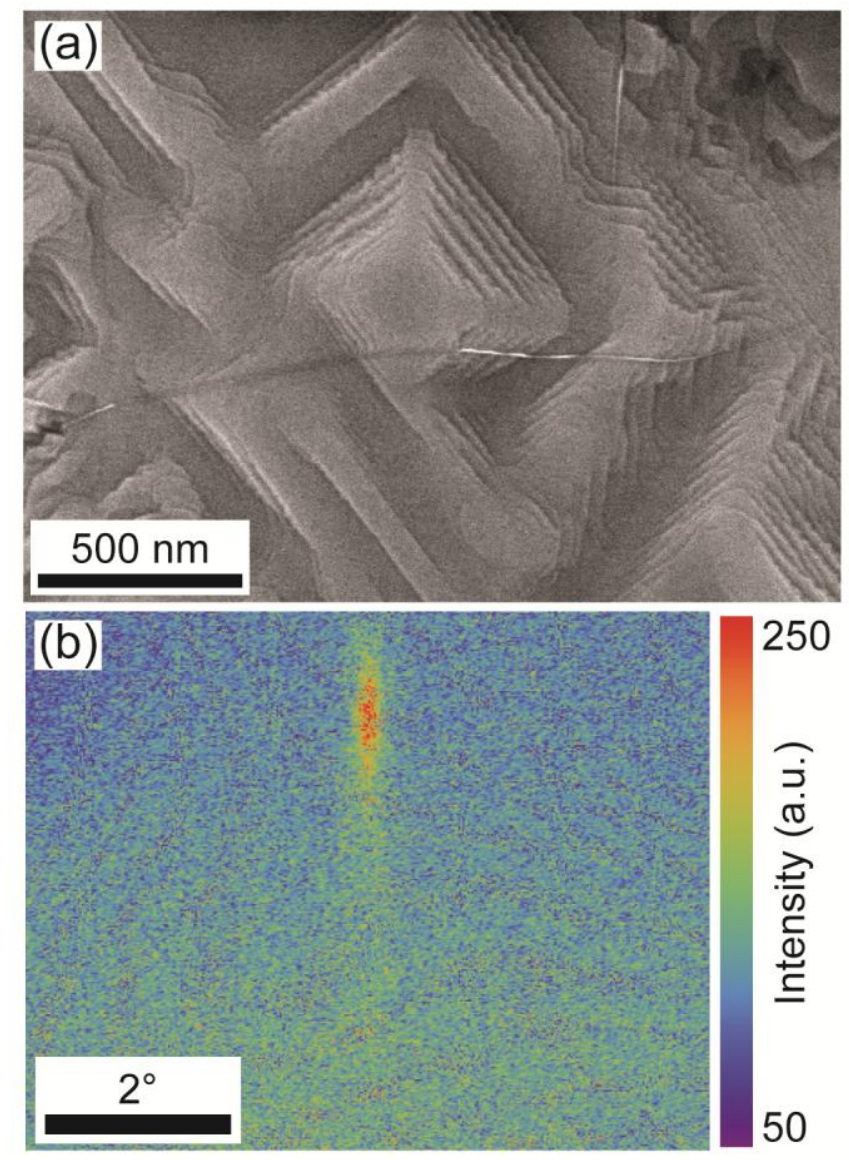

